\title{
Evidence of Griffiths Phase Behavior in Paramagnetic State of Heavy Fermion Compounds $\mathrm{Ce}_{x} \mathrm{La}_{1-x} \mathrm{~B}_{6}(0.01 \leq x \leq 1)$
}

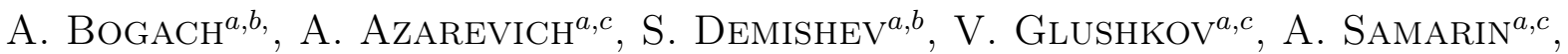 \\ N. Shitsevalova ${ }^{d}$, V. Filipov $^{d}$, S. GabÁni $^{e}$, K. Flachbart $^{e}$ And N. Sluchanko ${ }^{a, c}$ \\ ${ }^{a}$ Prokhorov General Physics Institute, RAS, Vavilov str. 38, 119991 Moscow, Russia \\ ${ }^{b}$ National University of Science and Technology "MISIS", Leninskiy pr.4, Moscow 119049, Russia \\ ${ }^{c}$ Moscow Institute of Physics and Technology, Moscow Region 141700 Russia \\ ${ }^{d}$ Institute for Problems of Materials Science, NASU, Krzhizhanovsky str., 3, 03142 Kyiv, Ukraine \\ ${ }^{e}$ Institute of Experimental Physics SAS, Watsonova 47, 04001 Košice, Slovakia
}

\begin{abstract}
We have investigated the doping-induced changes of magnetic properties of the archetypal heavy fermion compound $\mathrm{CeB}_{6}$ on a series of substitutional solid solutions $\mathrm{Ce}_{x} \mathrm{La}_{1-x} \mathrm{~B}_{6}(0.01 \leq x \leq 1)$. For these strongly correlated electron systems high precision measurements of magnetic susceptibility $\chi(T)$ were undertaken in small external magnetic field aligned along [100], [110], and [111] directions in the simple cubic lattice of these hexaborides. It was shown that for all crystals and all magnetic field orientations the $\chi(T)$ dependences obey a power law $\chi \sim T^{-\alpha}$ at temperatures between 10 and $200 \mathrm{~K}$. The observed critical exponents $\alpha=0.77-0.93$ demonstrate a monotonous decrease with the increase of Ce content. It is concluded that such a kind of Griffiths phase-type behavior that differs considerably from the convenient Curie-like dependence should be attributed to the emergence of disordered nanometer-size magnetic clusters of Ce-ions in the $\mathrm{RB}_{6}$ matrix.
\end{abstract}

DOI: 10.12693/APhysPolA.137.782

PACS/topics: nanometer magnetic clusters, Griffiths phase

\section{Introduction}

In the past decade investigations of spatial anisotropy that is generated spontaneously in the translationally invariant metallic phase, e.g., in the form of electron nematic or spin nematic effect, have addressed a great challenge for both experimentalists and theoreticians [1-2]. Indeed, numerous experimental objects like ultra clean quantum Hall systems, ruthenates, high- $T_{\mathrm{C}}$ superconductors [1], and iron-based superconductors [2-3] demonstrate unexpected anisotropy in their electronic properties, forbidden by the high symmetry of studied systems. As pointed out in [1], it looks amazing how point-like electrons demonstrate properties similar to those of liquid crystals, where rod-shaped molecules act as basic blocks to form a nematic phase. Up to now, several theoretical mechanisms were proposed to explain the electron nematic effect including Pomeranchuk instability of Fermi liquid, or melting of a stripe phase [1-3].

Another intriguing option for the realization of an electron nematic phase is provided by crystals with orbital ordering, as long as both orbitally ordered states and electron nematic phases may be considered as systems with broken spatial symmetry $[1,3]$. It was shown recently [4] that a possible candidate for the search of nematic effects related to orbital order is cerium

corresponding author; e-mail: alex@lt.gpi.ru hexaboride $\mathrm{CeB}_{6}$. This strongly correlated metal with a simple cubic lattice is believed to represent an archetypal dense Kondo system with small Kondo temperature $\left(T_{\mathrm{K}} \approx 1-2 \mathrm{~K}\right)$. This is, in fact, comparable with the temperatures of two magnetic phase transitions observed in $\mathrm{CeB}_{6}$ at $T_{Q} \approx 3.2 \mathrm{~K}$ (orbital ordering in the antiferroquadrupole $\mathrm{AFQ}$ phase) and at Néel temperature $T_{\mathrm{N}} \approx 2.3 \mathrm{~K}[4]$ (see the magnetic phase diagram in Fig. 1). Taking into account the loosely bound state of the rare earth (RE) atoms in the simple cubic crystal lattice [5], it is natural to expect random local displacements of rare-earth ions, and the formation of nanometer magnetic clusters in the $\mathrm{RB}_{6}$ matrix at low temperatures. Thus, the purpose of this study was to investigate the magnetization of a series of substitutional solid solutions $\mathrm{Ce}_{x} \mathrm{La}_{1-x} \mathrm{~B}_{6}(0.01 \leq x \leq 1)$ in the paramagnetic state. This sheds light on the features of magnetic response in the temperature range prior the emergence of the electron nematic phase.

\section{Experimental details}

The high quality single crystals of $\mathrm{Ce}_{x} \mathrm{La}_{1-x} \mathrm{~B}_{6}$ $(0.01 \leq x \leq 1)$ were grown by vertical crucible-free inductive floating zone melting in an inert gas atmosphere. The high quality of crystals was controlled by X-ray diffraction, Laue backscattering-patterns, and electron microprobe analysis. Magnetization between 1.8 and $300 \mathrm{~K}$, and in low magnetic field $(0.1-2 \mathrm{kOe})$ was studied by an MPMS-5 commercial SQUIDmagnetometer (Quantum Design). 


\section{Results and discussion}

Relevant contributions to magnetization $M(T)$ originate, on the one side, from the diamagnetic boron network and Pauli paramagnetic component $\left(\chi_{0}\right)$, while on the other side, from the temperature dependent magnetic response provided by weakly interacting magnetic moments of Ce ions. To separate those contributions, the data of magnetic measurements (see, e.g., in Fig. 1a) were plotted in the form of product $T M(T)$ (Fig. 1b). The linear slope of these dependencies was used to estimate the $\chi_{0}$ values for every $\mathrm{Ce}_{x} \mathrm{La}_{1-x} \mathrm{~B}_{6}$ composition. Figure 2 shows the temperature dependent magnetic susceptibility $\chi_{-} \chi_{0}$ obtained for 9 Ce-concentrations in the range between $x=0.01$ and $\mathrm{CeB}_{6}$, and for three principal directions of external magnetic field $\boldsymbol{H} \|[100]$, [110], and [111] (altogether 27 crystals were studied). From the double logarithmic plot in Fig. 2, it can be discerned that the power law of the magnetic susceptibility behavior is valid in a wide temperature range $10-200 \mathrm{~K}$ for all Ce-based hexaborides. As an example, a reciprocal susceptibility as a function of $T^{\alpha}(\alpha=0.79)$ for $\mathrm{CeB}_{6}$ is shown on the insert in Fig. 2.

To analyze the temperature dependent magnetic response, a simple formula was used

$$
\chi_{-} \chi_{0}=\left(\frac{C}{T^{\prime}}\right)\left(\frac{T^{\prime}}{T}\right)^{\alpha} .
$$

Note, that with $T^{\prime}=1 \mathrm{~K}$, which in the case if $\alpha=1$, (1) is equivalent to Curie-like behavior of susceptibility with a Curie constant $C=N \mu_{\text {eff }}^{2} /\left(3 k_{\mathrm{B}}\right)$, where $N$ is the number of magnetic sites per unit cell, $\mu_{\text {eff }}$ is their effective magnetic moment, and $k_{\mathrm{B}}$ is the Boltzmann constant. The set of fitting parameters in (1) obtained in the temperature range $10-200 \mathrm{~K}$, is presented in Fig. 3.

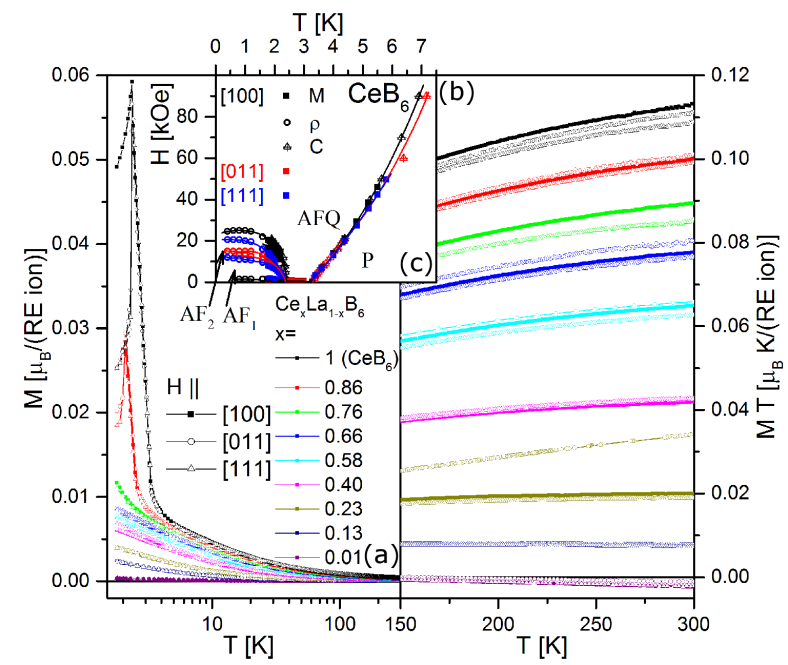

Fig. 1. Temperature dependencies of (a) magnetization, (b) the $M T$ product (see text) for $\mathrm{Ce}_{x} \mathrm{La}_{1-x} \mathrm{~B}_{6}$, and (c) the magnetic phase diagram of $\mathrm{CeB}_{6} . \mathrm{AF}_{1}$, $\mathrm{AF}_{2}$, and $\mathrm{AFQ}$ denote antiferromagnetic and $\mathrm{P}-$ paramagnetic phases.

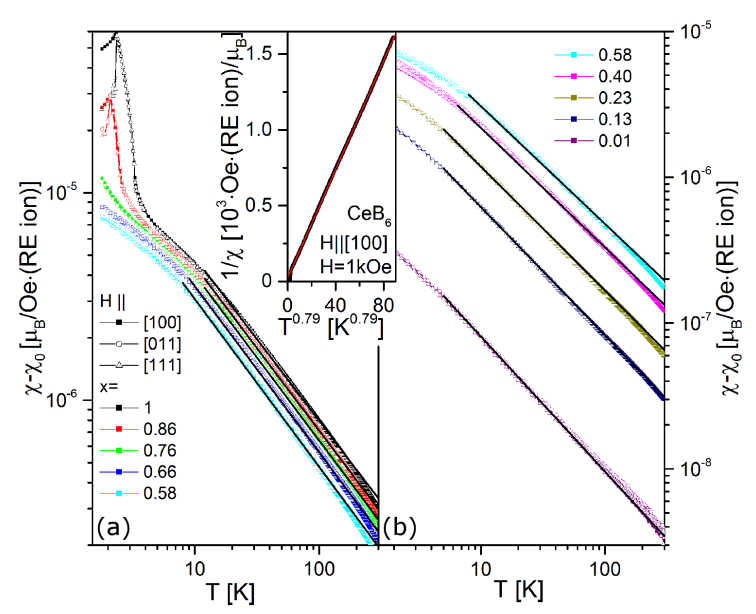

Fig. 2. Temperature dependencies of magnetic susceptibility $\chi-\chi_{0}$ for $\mathrm{Ce}_{x} \mathrm{La}_{1-x} \mathrm{~B}_{6}$ crystals with (a) $0.5<$ $x<1$, and (b) $0.01 \leq x<0.6$. Inset shows the data plot $1 / \chi\left(T^{\alpha}\right)$ for $\mathrm{CeB}_{6}$.

It is worth noting that approximately linear dependence of $\chi_{0}(x)$ is observed in $\mathrm{Ce}_{x} \mathrm{La}_{1-x} \mathrm{~B}_{6}$ hexaborides which may be explained in terms of renormalization of the electron density of states (DOS) due to a strong increase of the Pauli-like temperature independent susceptibility component of these Ce-based heavy fermion compounds. We note also that the initial negative values of $\chi_{0}(x)$ in metallic $\mathrm{Ce}_{x} \mathrm{La}_{1-x} \mathrm{~B}_{6}$ solid solutions are caused by diamagnetic properties of the rigid boron network [5]. It is easy to see from Fig. 3 b that the inequality $\alpha<1$ is valid for all studied Ce hexaborides, and the observed critical exponents $\alpha=0.77-0.93$ demonstrate a monotonous decrease with the increase of Ce content.

Such a kind of Griffiths phase-type behavior [6-8] is very different from the convenient Curie-like dependence and should be attributed to the emergence of disordered magnetic clusters of Ce-ions in the matrix of hexaborides. Taking into account the loosely bound state of RE atoms in the simple cubic crystal lattice [5], it is natural to conclude that the random local displacements of rare-earth ions from central positions in the $B_{24}$ cubooctahedrons lead to formation of nanometer-size magnetic clusters in the $\mathrm{RB}_{6}$ matrix. The power law of susceptibility (1) in the Griffiths phase consisting of spin clusters can be naturally explained in terms of different values of exchange integral $J[6-8]$, which in turn manifests itself as a power distribution function of exchange energies and reflects the low temperature correlations in the spin system. Taking into account that antiferromagnetic correlations are dominating in $\mathrm{CeB}_{6}[9]$ one may suppose the presence of AF Ce-dimers in the $\mathrm{RB}_{6}$ matrix with $\mathrm{AF}$ exchange. Indeed, taking into account random distribution of $\mathrm{Ce}$ ions in matrix of $\mathrm{Ce}_{x} \mathrm{La}_{1-x} \mathrm{~B}_{6}$ solid solutions it is natural to expect a formation of Ce-Ce pairs already for $x>0.05$. A probability analysis in the framework of simple relation

$$
n_{d}=n_{\mathrm{Ce}}\left[1-\left(1-n_{\mathrm{Ce}}\right)^{z}\right],
$$




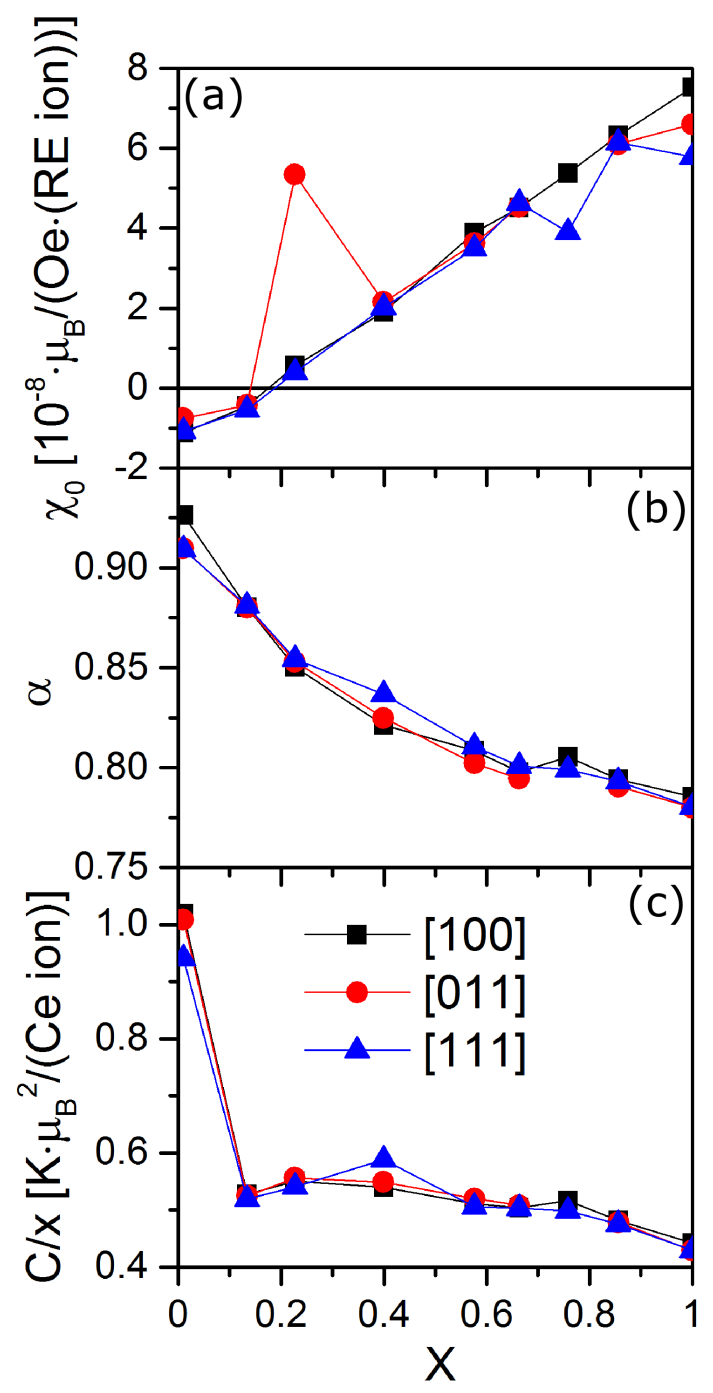

Fig. 3. Concentration dependencies of the fitting parameters: (a) temperature independent susceptibility $\chi_{0}$, (b) critical exponent $\alpha$, and (c) normalized $C / x$ ratio (see (1))

that connects the Ce content $n_{\mathrm{Ce}}$ and concentration of Ce-dimers $n_{d}$ (coordination number $z=6$ for a simple cubic lattice of $\mathrm{R}$ ions), leads to the value $n_{d} \approx 0.075$ for $\mathrm{Ce}_{0.13} \mathrm{La}_{0.87} \mathrm{~B}_{6}$. Thus, Ce-dimers are with a high probability formed, and they dominate in hexaborides. Very recently it was shown that the properties of $\mathrm{GdB}_{6}$ are determined by two factors, (i) by vibrationally coupled Gd-dimers, and (ii) by dynamic charge stripes that are responsible for the dramatic symmetry lowering with temperature decrease [10]. We propose that such a scenario is common for the rare earth hexaborides, and that the formation of the Griffiths phase shown here for $\mathrm{Ce}_{x} \mathrm{La}_{1-x} \mathrm{~B}_{6}$ compounds at intermediate temperatures precedes the electron nematic effect developed in the AFQ state of $\mathrm{CeB}_{6}$.

\section{Conclusions}

Precise magnetic measurements on a series of substitutional solid solutions $\mathrm{Ce}_{x} \mathrm{La}_{1-x} \mathrm{~B}_{6}(0.01 \leq x \leq 1)$ have been carried out at temperatures $1.8-300 \mathrm{~K}$ in an external magnetic field aligned along [100], [110] and [111] directions in the simple cubic lattice of these hexaborides. The Griffiths phase-type behavior $\chi \sim T^{-\alpha}$ with critical exponents $\alpha=0.77-0.93$ was found at temperatures in the range $10-200 \mathrm{~K}$ for all single crystals and for all orientations of magnetic field. We argue that this kind behavior should be attributed to the formation of disordered nanometer-size spin clusters of Ce ions with AF exchange in combination with strong effects on the DOS renormalization of the paramagnetic Pauli susceptibility component in these Ce-based heavy fermion compounds.

\section{Acknowledgments}

This work was supported by the Russian Science Foundation, project No. 17-12-01426 and Slovak agencies VEGA (grant No. 2/0032/16) and APVV (grant No. 17-0020).

\section{References}

[1] E. Fradkin, S.A. Kivelson, M.J. Lawler, J.P. Eisenshtein, A.P. Mackenzie, Annu. Rev. Condens. Matter Phys. 1, 153 (2010).

[2] J.H. Chu, J.G. Analytis, K. De Greve, P.L. McMahon, Z. Islam, Y. Yamamoto, I.R. Fisher, Science $\mathbf{3 2 9}$ 824 (2010).

[3] R.M. Fernandes, A.V. Chubukov, J. Schmalian, Nat. Phys. 10, 97 (2014)

[4] S. V. Demishev, V.N. Krasnorussky, A.V. Bogach, V.V. Voronov, N. Yu. Shitsevalova, V.B. Filipov, V.V. Glushkov, N.E. Sluchanko, Sci. Rep. 7, 17430 (2017).

[5] K. Flachbart, P. Alekseev, G. Grechnev, N. Shitsevalova, K. Siemensmeyer, N. Sluchanko, O. Zogal, in: Rare Earths: Research and Applications, Ed. K.N. Delfrey, Nova Science Publishers, New York 2008, p. 79.

[6] R.B. Griffiths, Phys. Rev. Lett. 23, 17 (1969).

[7] A.J. Bray, Phys. Rev. Lett 59, 586 (1987).

[8] S. Demishev, Phys. Stat. Sol. B 247, 676 (2010).

[9] N.E. Sluchanko, A.V. Bogach, V.V. Glushkov et al. J. Exp. Theor. Phys. 104, 120 (2007).

[10] A.P. Dudka, O.N. Khrykina, N.B. Bolotina, N.Yu. Shitsevalova, V.B. Filipov, M.A. Anisimov, S. Gabani, K. Flachbart, N.E. Sluchanko, Phys. Rev. B 100, 205103 (2019). 